\title{
Uterine and placental KISS1 regulate pregnancy: what we know and the challenges that lie ahead
}

\author{
Andy $\vee$ Babwah ${ }^{1,2,3}$ \\ ${ }^{1}$ The Children's Health Research Institute, ${ }^{2}$ Lawson Health Research Institute, and ${ }^{3}$ Departments of Obstetrics and \\ Gynaecology and Physiology and Pharmacology, The University of Western Ontario, 800 Commissioners Road East, \\ London, Ontario, Canada N6C 2V5
}

Correspondence should be addressed to A V Babwah; Email: ababwah@uwo.ca

\begin{abstract}
Hypothalamic KISS1 and its derivatives (kisspeptins) are now well recognized as potent stimulators of GnRH secretion and thereby major regulators of the neuroendocrine-reproductive axis. Recent studies in the mouse strongly suggest that independent of the hypothalamus and pituitary, peripherally derived KISS1 also regulates fertility, and disruption of local KISS1 signaling in the ovary and uterus is sufficient to trigger infertility. With this increasing recognition that peripherally derived KISS1 regulates fertility, the first goal of this review is to critically discuss the data that have led to this conclusion, focusing on uterine- and placental-derived KISS1. Given that a significant amount of this data was generated in animals such as the mouse and rat, a second goal of this review is to identify and discuss the limitations of the animal data in the context of better understanding KISS1 as a regulator of human pregnancy. The growing evidence suggests that in both man and mouse, KISS1 plays an important role in regulating very early pregnancy events such as embryo implantation. However, as pregnancy advances, although it seems that KISS1 continues to play important roles in regulating human pregnancy, it might not do so in the mouse. This surprising functional dichotomy between human females and mice appears also to exist between women and a large number of animal species, including lower primates. These findings are of tremendous significance and will greatly shape how KISS1 will be developed as a therapeutic agent in augmenting the reproductive potential of both women and important livestock species.

Reproduction (2015) 150 R121-R128
\end{abstract}

\section{Introduction}

Centrally derived KISS1 (the 145 amino acid primary translation product of the KISS1 gene) and its derivatives (kisspeptins) are now well recognized as potent triggers of hypothalamic gonadotropin-releasing hormone $(\mathrm{GnRH})$ secretion and thereby serve as pivotal regulators of the neuroendocrine-reproductive axis (reviewed in Millar \& Babwah (2015)). Diminished hypothalamic kisspeptin signaling in man and mouse, as a consequence of inactivating mutations in the genes that encode either KISS1 or its cognate receptor, KISS1R, results in infertility. Initially, this infertility was thought to be the result of congenital hypogonadotropic hypogonadism, a disorder that originates at the level of the hypothalamus and/or pituitary and is characterized by the absence of spontaneous sexual maturation in the presence of low gonadotropin luteinizing hormone and follicle-stimulating hormone and sex steroids (estradiol $\left(E_{2}\right)$ and testosterone) in the absence of anatomical or functional abnormalities of the hypothalamic-pituitary-gonadal axis. However, recent studies in the mouse strongly suggest that, independent of the hypothalamus and pituitary, a disruption of local KISS1 signaling in the ovary or uterus can also trigger infertility (Calder et al. 2014, Gaytan et al. 2014). These important findings further strengthen human data that peripherally derived KISS1 regulates pregnancy (Janneau et al. 2002, Horikoshi et al. 2003, Bilban et al. 2004, Taylor et al. 2014). With the growing appreciation that local kisspeptin is a major regulator of pregnancy, my first objective is to review and critically discuss the data that have led to such an appreciation while focusing on uterine- and placental-derived KISS1. Given that a significant amount of the data that has considered or examined the roles of peripherally derived KISS1 in regulating fertility and pregnancy were derived from animals such as the mouse and rat, a second goal of this review is to discuss the limitations of the animal data with respect to better understanding KISS1 as a regulator of human pregnancy.

\section{Early evidence suggesting that peripheral KISS1 regulates human placentation}

When KISS1 was first discovered and reported to act as a metastatic suppressor of melanoma cells, it was also reported, based on northern blot analyses, that KISS1 
was highly expressed in the normal human placenta (Lee et al. 1996). This was the first evidence that KISS1 might regulate human placentation. Janneau et al. (2002) confirmed by RT-PCR that KISS1 and KISS1R are expressed in the human placenta and that their expression was much greater in the highly invasive early placenta (7-9 weeks) compared to the noninvasive term placenta (39-41 weeks). Additionally, expression levels were significantly greater in benign trophoblast tumors associated with hydatidiform moles (nonmetastatic malignant tissues isolated at 9-12 weeks) compared to choriocarcinoma cell lines ranging from the weak metastatic BeWo and JAR cells to the more invasive JEG-3 cells (Grümmer et al. 1994, Morgan et al. 1998, Xu et al. 2001). The implication of these findings is that in healthy pregnancies KISS1 and KISS1R inhibit the invasive and migratory properties of trophoblast cells and thus the placenta-based kisspeptin/KISS1R signaling system is a regulator of human placentation. The finding was consistent with the proposed role for KISS1 in inhibiting the metastatic potential of malignant melanoma cells in that, among a panel of related malignant melanoma cell lines, KISS1 was only expressed in the nonmetastatic cell line and was undetectable even among lines that showed low metastatic potential (Lee et al. 1996).

In a study designed to identify molecules that regulate trophoblast invasion, using gene microarrays, Bilban et al. (2004) compared the mRNA expression profiles between trophoblasts derived from highly invasive first trimester and poor to noninvasive term placentae. This study identified 643 differentially expressed genes displaying greater than a twofold difference between the two populations of trophoblasts. The expression of genes from the invasive first trimester trophoblasts was further compared to the expression of genes from the poorly invasive but proliferative BeWo trophoblast cell line (Grümmer et al. 1994, Morgan et al. 1998, Xu et al. 2001). Genes that were differentially expressed in the invasive first trimester trophoblasts were viewed as putative regulators of invasion. KISS1, which had displayed a 29-fold higher expression level in first trimester trophoblasts compared to term trophoblasts, was among the 220 candidate genes.

Clearly, based on the studies by Lee et al. (1996), Janneau et al. (2002) and Bilban et al. (2004), it seemed likely that KISS1 plays an important role in regulating trophoblast invasion during early placentation. Regulation of the invasive and migratory properties of trophoblast cells is critical to normal placentation. Excessive or reduced invasion and migration of trophoblasts during placentation have pathological consequences; thus, these processes are highly regulated by factors that either promote or inhibit migration and invasion.

\section{Kisspeptin negatively regulates trophoblast migration and invasion}

While the findings reported by Janneau et al. (2002) strongly suggested that KISS1 negatively regulates human trophoblast migration and invasion, it was the studies of Bilban et al. (2004) that provided the first experimental evidence that it did. After demonstrating the expression of KISS1 within the syncytiotrophoblast and KISS1R within both villous (syncytiotrophoblast and cytotrophoblast) and extravillous trophoblasts (EVTs), the authors demonstrated that exogenous kisspeptin, without affecting cell viability and proliferation, significantly inhibited EVT outgrowth from cultured villi explants (from 6- to 9-week-old placentae). Similarly, exogenous kisspeptin, without affecting proliferation, also inhibited EVT migration and invasion of either fibronectin or collagen $\mathrm{I}$ in transwell assays. Both fibronectin and collagen are major components of the human placenta (Amenta et al. 1986, van der Rest \& Garrone 1991). Taken together, the data suggest that kisspeptin negatively regulates EVT migration and invasion. Bilban et al. (2004) also determined that mechanistically, kisspeptin suppressed trophoblast invasion by inhibiting trophoblast MMP2 proteolytic activity, thus reducing the capacity of EVTs to degrade and invade the surrounding matrix.

Further mechanistic insight into how kisspeptin negatively regulates EVT migration and invasion came from a study conducted in my laboratory (Taylor et al. 2014) using HTR-8/SVneo placental cells expressing exogenous human KISS1R. The parental HTR-8/SVneo line is an established and highly invasive human EVT cell line derived from a first trimester placenta (Graham et al. 1993). Here we demonstrated that kisspeptin, in a dosedependent manner, signals via ERK $1 / 2$ and protein kinase $C$ to rapidly increase the adhesion of EVTs to type I collagen. We therefore suggested that in addition to inhibiting MMP2 activity, kisspeptin further restricts EVT migration and invasion to regulate normal placentation by increasing its adhesiveness to its substrate. However, whether these two kisspeptin-regulated events are mechanistically linked remains to be determined. Additionally, we demonstrated that kisspeptin increased the adhesion of hatched mouse blastocysts to type I collagen, suggesting that kisspeptin is not just a regulator of trophoblast function during early placentation but also a regulator of the preceding implantation event as well.

\section{Kisspeptin positively regulates embryo implantation}

In assisted reproduction, $\sim 50 \%$ of transferred embryos fail to implant in the mother's uterus despite these embryos appearing to be healthy. Not surprisingly, implantation remains the least understood key ratelimiting step in assisted reproductive technology (Boomsma et al. 2009). Implantation is comprised of 
three steps; apposition, adhesion, and penetration. Based on our finding that kisspeptin increases the adhesion of mouse blastocysts to type I collagen, we hypothesized that kisspeptin positively regulates embryo implantation and proceeded to test this using the Kiss 1 and Kiss1r null mice. Here we found that in hormoneprimed null females, following mating to WT males, Kiss $1^{+/-}$embryos failed to implant in their Kiss $1^{-1-}$ mothers even as late as day 7 (D7) of pregnancy. We clearly demonstrated that while normal embryo apposition occurred, embryo adhesion and penetration did not (Calder et al. 2014), fully supporting our hypothesis.

Next, we demonstrated that Kiss $1^{+/-}$embryos, which failed to implant in the Kiss $1^{-1-}$ uterus, implanted in the uterus of a WT recipient female; thus, the inability to implant was due to a maternal and not embryonic defect. Additionally, because exogenous gonadotropins, $E_{2}$ and progesterone could not rescue the implantation failure, we concluded that the defect was uterine based (Calder et al. 2014) and considered that it was the lack of a functional uterine-based kisspeptin signaling system in the null female that disrupted embryo implantation. In support of this idea, we found that on the day of implantation (D4 of pregnancy), the WT mouse uterus expresses kisspeptin and Kiss1r, a finding also made by Zhang et al. (2014) based on both RT-PCR and immunohistochemical analyses. More importantly, we also demonstrated that at this critical peri-implantation period, the WT uterus expresses a functional kisspeptin/ KISS1R signaling system localized to both the luminal and glandular epithelia (Fig. 1). Thus, in the Kiss $1^{-1-}$ and $K_{i s s} 1 r^{-1-}$ uterus, this signaling system would be disrupted on the day of implantation.

Could this uterine-based kisspeptin/KISS1R signaling system be mediating embryo adhesion to the luminal epithelium? We considered that possibility and on further investigation, through immunostaining, found that the $\mathrm{KisS1}^{-1-}$ endometrium exhibited highly reduced levels of the glandular leukemia inhibitory factor (LIF). LIF is a member of the interleukin 6 cytokine family, and glandular LIF has been demonstrated to be absolutely essential for blastocyst implantation in the mouse (Bhatt et al. 1991, Stewart et al. 1992). Based on in situ analyses, Lif exhibits a biphasic expression pattern in the mouse uterus on D4 of pregnancy. In the morning it is expressed in the glandular epithelium and in the evening it is expressed in stromal cells at the site of embryo attachment (Song et al. 2000, Song \& Lim 2006). These observations suggest that on D4, LIF has two roles: first, in the preparation of the uterus, and second, in the attachment of the embryo to the endometrial lining.

Interestingly, in the Lif $^{-1-}$ female, Lif $^{-1-}$ blastocysts also undergo normal apposition and make contact with the endometrial lining but do not undergo adhesion because they could be flushed out of the uterus on D7 of pregnancy (Stewart et al. 1992, Chen et al. 2000). We therefore tested whether LIF was a limiting factor in the

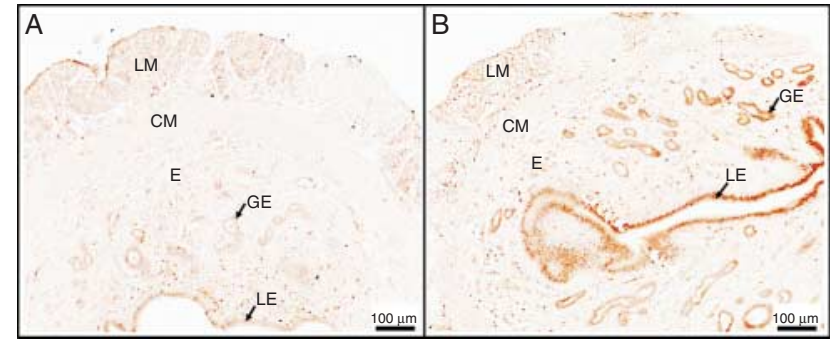

Figure 1 Phosphorylated p38 levels increase in the uteri of KP54-treated pregnant WT female mice. Eight- to 12-week-old WT female and male $129 S 1 / S v I m J$ mice were mated, and on the morning of D4 of pregnancy, females were injected with either PBS $(100 \mu \mathrm{l})$ or KP54 $(100 \mathrm{nmol}$ $\mathrm{KP} 54 / \mathrm{kg}, 100 \mu \mathrm{l}$ ) intraperitoneally and were sacrificed $30 \mathrm{~min}$ later. The uteri were removed and simultaneously processed for immunohistochemistry using standard techniques (Calder et al. 2014) and $5 \mu \mathrm{m}$-thick slices were simultaneously immunostained to detect phosphorylated p38. Care was taken to expose the experimental and control tissue to identical experimental conditions. Phosphorylated p38 was detected with the phospho-p38 MAPK (Thr180/Tyr182) (D3F9) XP Rabbit mAb (Cell Signaling, Boston, MA, USA) in conjunction with the ImmunoCruz rabbit ABC Staining System sc-2018 (Santa Cruz Biotechnology, Inc.). Both the primary and secondary detection systems were used according to the manufacturers' guidelines without any adaptations. Results show (a highly representative image from an 8-week-old pregnant female) that, compared to PBS-treated mice (A), the uteri from KP54-treated mice (B) exhibited higher levels of phosphorylated p38 in both the LE and GE. These findings were recapitulated when uteri were removed from untreated mice and then treated with either PBS or KP54 in vitro in the absence of any effects that KP54 might have triggered at the level of the hypothalamus, pituitary, and ovaries (data not shown). Overall, the data reveal that in the mouse uterus on D4 of pregnancy, there is a functional kisspeptin/KISS1R signaling system. LM, longitudinal muscle of the myometrium;

$\mathrm{CM}$, circular muscle of the myometrium; $\mathrm{E}$, endometrium; LE, luminal epithelium; GE, glandular epithelium. Scale bar $=100 \mu \mathrm{m}$.

Kiss $1^{-1-}$ uterus and found that exogenous LIF was indeed sufficient to partially rescue implantation (Calder et al. 2014). Based on these findings we suggested that uterinebased KISS1 signaling, which is disrupted in the Kiss $1^{-1-}$ uterus, promotes glandular LIF expression and availability and thereby regulates embryo implantation.

In the mouse uterus, Lif expression is regulated by estrogen. This is based on the observations that the expression of uterine Lif is upregulated at ovulation and that $L i f$ is not expressed in the uterus during experimentally induced delayed implantation but is rapidly induced following an injection of estrogen (Bhatt et al. 1991, Song et al. 2000). Using an RNase protection assay, Chen et al. (2000) confirmed that estrogen rapidly upregulates uterine Lif expression and further demonstrated that LIF can replace nidatory estrogen at inducing both implantation and decidualization in ovariectomized mice. Interestingly, in the Kiss ${ }^{-1-}$ mouse, whereas LIF could partially rescue implantation failure, $E_{2}$ was unable to do so (Calder et al. 2014). Failure of $\mathrm{E}_{2}$ to rescue implantation in this mouse unlikely reflects a technical limitation because $E_{2}$ administered in an identical manner has successfully rescued delayed implantation events 
(reviewed in Dey et al. (2004)). Instead, it suggests that KISS1 might either lie downstream of $\mathrm{E}_{2}$ in triggering Lif expression or that KISS1 and $\mathrm{E}_{2}$ are obligate coregulators of Lif expression. Based on recent data from Zhang et al. (2014), it appears that KISS1 lies downstream of $E_{2}$ because the authors showed, through the use of RT-PCR, that in the ovariectomized mouse uterus the expression of Kiss 1 mRNA was upregulated after $E_{2}$ treatment. This finding is important and suggests that $\mathrm{E}_{2}$-regulated Kiss1 expression, which is well documented in the hypothalamus (reviewed in Pinilla et al. (2012)), is conserved in the uterus.

\section{Kisspeptin positively regulates endometrial decidualization}

Chen et al. (2000) reported that i.p. injections of LIF on D4 or D5 of pregnancy was sufficient to induce a state of receptivity in the uterus, permitting embryo implantation and decidualization. Furthermore, these pregnancies resulted in live births leading the authors to conclude that the transient expression of LIF at the uterine epithelium is sufficient for blastocyst implantation and decidualization and that LIF is not required for postimplantation development or for the maintenance of pregnancy. While in our studies D4 LIF-treated pregnant Kiss $1^{-1-}$ females were only examined up to D10 of pregnancy, we too found that up to that day the pregnancy appeared to have proceeded normally.

If KISS1 lies upstream of LIF and regulates glandular LIF availability, it would be reasonable to conclude that transient KISS1 expression on D4 of pregnancy would also be sufficient to trigger both implantation and decidualization. However, Zhang et al. (2014) found that the expression of Kiss1 and Kiss1r in the mouse uterus was not transiently restricted to the periimplantation period. Instead, they found that between D5 and D8 the expression of these molecules increased significantly in the pseudopregnant uterus during artificially induced decidualization. Similar findings were also made in the uterus of the pregnant mouse, and in both cases (artificial decidualization and true pregnancy) the levels of KISS1 and KISS1R proteins and mRNA expression were similar. The authors recapitulated these in vivo findings in a stromal cell culture model. Here, they reported that the expression of Kiss 1 and Kiss1rincreased with the progression of stromal cell decidualization and that the process was blocked by downregulating Kiss1 expression using siRNA against Kiss1. Taken together, these results strongly suggest a role for KISS1 in inducing and maintaining decidualization of the mouse endometrium, at least during early pregnancy.

Decidualization of the mouse uterus begins with embryo implantation at the antimesometrial pole of the uterus. This primary decidualization that occurs on D4 of pregnancy is then followed by a wave of secondary decidualization, which begins antimesometrially, progresses laterally and then mesometrially gives rise to the decidua basalis. From D5 to D8 of pregnancy, most of the tissue at the site of implantation is maternal decidua. However, decidua is a transient tissue, and starting at around D9 of pregnancy, the antimesometrial decidua begins to regress and by D11 all that remains is a very thin layer; however, during pregnancy, the mesometrial decidua basalis persists (reviewed in Ramathal et al. (2010) and Pang et al. (2014)).

When the studies by Chen et al. (2000), Calder et al. (2014) and Zhang et al. (2014) are considered together, the question that emerges is whether KISS1 expression on D4 of pregnancy is sufficient for inducing and maintaining decidualization by increasing glandular LIF levels or whether KISS1 expression is required postimplantation to trigger secondary decidualization as well? Collectively, the studies by Chen et al. (2000) and Calder et al. (2014) suggest it is because a single injection of LIF was able to rescue implantation on D4 as well as induce and maintain decidualization beyond this point. However, the study by Zhang et al. (2014) suggests that it is not because uterine Kiss1/KISS1 expression beyond D4 appeared necessary for maintaining decidualization. Clearly, more detailed investigations will be required to better understand the relevance of the postimplantation endometrial expression of the KISS1/ KISS1R signaling system in the mouse uterus.

\section{Are the pregnant mouse and rat adequate animal models to study human pregnancy?}

While the mouse and rat are invaluable animal models for studying numerous biological processes under both normal and pathological conditions in man, it is important to consider the limitations of these models in a given process. Since the studies of Janneau et al. (2002) and Bilban et al. (2004), which gave us our first convincing reasons to believe that placental-derived KISS1 could regulate human placentation, there has been growing excitement in further studying the roles of peripheral KISS1 in regulating not just human placentation but also human pregnancy in general, and some of these studies (reviewed in Bhattacharya \& Babwah (2015)), including those from my laboratory (Calder et al. 2014, Taylor et al. 2014), have been initiated in the mouse and rat. However, several observations have been made that clearly suggest that there are striking differences in how KISS1 regulates pregnancy between these two laboratory animals and human females. The following sections highlight these putative and established differences.

\section{Does peripheral KISS1 regulate human embryo implantation?}

The discrete steps in implantation - that is, embryo apposition, adhesion, and penetration - are the same in 
both mouse and man. Thus, it is likely that these processes may be similarly regulated. KISS1 and KISS1R are expressed in the human endometrium in both the luminal and glandular epithelia throughout the menstrual cycle (Cejudo Roman et al. 2012). Based on this expression pattern, it is possible that the KISS1/ KISS1R signaling system regulates embryo implantation in the human uterus. If it does, it may also do so via LIF. In humans, LIF and its downstream signaling machinery are expressed in the secretory endometrium; in particular, LIF is highly expressed in the glandular epithelium and its levels are maximal in the secretory endometrium, suggesting the importance of LIF signaling in human pregnancy (Cullinan et al. 1996, Vogiagis et al. 1996, Hambartsoumian 1998). Additionally, LIF deficiency has been associated with unexplained recurrent abortions and infertility in women (Hambartsoumian 1998). If LIF does in fact regulate implantation in the human uterus, it is conceivable that both KISS1 and $\mathrm{E}_{2}$ might regulate its expression because the maximal period of implantation in human females (D20-D24 of the menstrual cycle) corresponds to elevated $E_{2}$ levels. However, this idea requires further investigation because data suggest that this mid-luteal rise in estrogen is not essential for successful implantation in women (reviewed in Groothuis et al. (2007)).

\section{Does peripheral KISS1 regulate human endometrial decidualization?}

In response to rising estrogen, epithelial and stromal cells in both mouse and man undergo extensive proliferation, and then in response to progesterone, stromal cells differentiate into decidual cells. In human females, the decidualization of stroma cells begins before the embryo implants during the late secretory phase; however, in the mouse, decidualization only occurs in response to implantation (reviewed in Dey et al. (2004)). Although the temporal difference appears minor, it might reflect other significant differences in decidualization between mouse and man. One possible difference might lie in the role of KISS1 in regulating decidualization as suggested by the following observation. During the first trimester of human pregnancy (specifically weeks 6-10 of gestation), based on both RT-PCR and immunofluorescence analyses, KISS1 and KISS1R were found to be strongly expressed in the placenta with KISS1 in the syncytiotrophoblast (Bilban et al. 2004) and possibly villous and extravillous cytotrophoblast (Francis et al. 2014) and KISS1R within both villous (syncytiotrophoblast and cytotrophoblast) and EVTs; their expression was not detected in decidualized stromal cells (Bilban et al. 2004). This finding may initially suggest that KISS1 does not regulate decidualization of the human endometrium. However, because the observations were made on 6- to 10-weekold placental tissue, we cannot rule out the possibility that the KISS1 signaling system is expressed in decidualizing cells at an earlier time point and that KISS1 regulates early decidualization of the human endometrium. Furthermore, in a recent study Wu et al. (2014) examined KISS1 and KISS1R expression in first trimester (approximately week 8 of gestation) decidua from women who miscarried a genetically normal fetus and from women who had a voluntary abortion and reported that while KISS1 expression was significantly reduced in the miscarried pregnancies, KISS1R levels were unchanged. The reasons for the contrasting data between the Wu et al. (2014) study and the Bilban et al. (2004) study are unclear but highlight the need for additional investigations.

\section{Does peripheral KISS1 regulate human myometrial contraction?}

In rodents, the myometrium, which encloses the endometrium, is composed of two distinct layers of smooth muscle: an inner circular layer and an outer longitudinal layer that is covered by peritoneum (serosa; Fig. 1). In humans, the general uterine muscle architecture is more complex than in rodents and is not composed of well-defined circular or longitudinal layers. Instead the muscular portion of the human uterus is composed of smooth muscle fibers organized into bundles, which in turn are organized into fascicles. The main function of the myometrium is to induce uterine contractions and facilitate expulsion of the fetus at parturition. Myometrial contractions are largely under the control of oxytocin (Phaneuf et al. 1995, Arrowsmith \& Wray 2014), and as a pregnancy approaches term, the number of oxytocin receptors increase significantly in the human myometrium (Kimura et al. 1996). Oxytocin is synthesized by hypothalamic supraoptic and paraventricular neurons and is released from the posterior pituitary gland into the circulation. As early as 2001, it was reported that i.v. administration of kisspeptin to female rats increases plasma oxytocin levels (Kotani et al. 2001) while more recently, Scott \& Brown (2011, 2013) reported that in nonpregnant and pregnant female rats, intravenously administered kisspeptin increased the firing rate of oxytocin neurons. Further investigation revealed that this peripheral effect was likely mediated via vagal afferents. Thus, it appears that in the rat, peripheral kisspeptin might regulate myometrial contractions via oxytocin and its receptor.

As Scott \& Brown (2011) pondered on whether their results observed in female rats would be recapitulated in women, they considered the following. In pregnant women the levels of kisspeptin (thought to be placenta derived) increase dramatically from 1000-1500 fmol/ml in the first trimester to $8000-11000 \mathrm{fmol} / \mathrm{ml}$ in the third trimester and stay elevated until parturition (Horikoshi et al. 2003). If kisspeptin levels also increase in the pregnant rat and remain chronically elevated, then in 
late pregnancy kisspeptin signaling should be desensitized (Pampillo et al. 2009, reviewed in Millar \& Babwah (2015)) and KISS1R would be unresponsive to exogenously administered kisspeptin (this topic is discussed further in Bhattacharya \& Babwah 2015). Yet, in their studies Scott \& Brown (2013) did not detect an attenuation in the effectiveness of exogenous kisspeptin on oxytocin neuron excitability in pregnant rats. The authors therefore considered that in the rat kisspeptin levels likely do not increase with the progression of pregnancy. But in women where kisspeptin levels do increase, does it mean that placental-derived kisspeptin plays no role in regulating myometrial contractions via oxytocin? Further studies are required to address this fascinating question.

Based on my personal communication with Dr Alain Caraty (2015) it is of tremendous importance to add that in addition to the rat, peripheral KISS1 levels were found to be very low and did not increase during pregnancy in the sheep, cow, pig, rabbit, horse, rhesus monkey, and marmoset (lower primates). It was unlikely that the failure to detect kisspeptin in these animals was technically based because the same reagents that included two validated antibodies readily detected plasma kisspeptin in pregnant women. Additional studies by Dr Alain Caraty (personal communication) demonstrated that the peripheral kisspeptin that they detected in pregnant women is bioactive and this bioactivity increased during gestation. Based on their unpublished findings, Dr Alain Caraty et al. have proposed that the increase in kisspeptin across gestation might be unique to women and possibly other higher female primates.

\section{Does peripheral KISS1 regulate human placentation?}

As stated earlier, based on studies using human placental tissues and cell lines (Janneau et al. 2002, Horikoshi et al. 2003, Bilban et al. 2004, Taylor et al. 2014) as well as the unpublished data from Dr Alain Caraty, there is compelling evidence to believe that KISS1 plays a significant role in regulating human placentation. The idea that it could do the same in mice and rats was advanced by the finding that both Kiss1 and Kiss1r, which were expressed in the trophoblast giant cells of the rat placenta at embryonic day 12.5 , gradually decreased during placental maturation leading the authors to suggest that, in the rat, KISS1/KISS1R signaling might regulate implantation of the mammalian embryo, placenta formation and maintenance of pregnancy (Terao et al. 2004).

In a recent study, Herreboudt et al. (2015) raised the strong possibility that KISS1 might not regulate placenta growth and development in rats and mice. The authors reported that they did not observe any differences in structure or in amino acid and glucose transport across the placentas of $K i s s 1^{-1-}$ and Kiss $1^{+/+}$placentas and thus concluded that kisspeptin signaling does not play a major role in placental development and function in the mouse. The finding was further supported by the very low levels of Kiss 1 expression found in E10.5 and E15.5 WT placenta. It is important to note, as the authors clearly stated, because the Kiss $1^{-1-}$ placentas were derived from heterozygous Kiss ${ }^{+/-}$females that were mated with $K i s s 1^{+/-}$heterozygous males, the Kiss $1^{-1-}$ placentas could have been rescued (in an endocrine and paracrine manner) by endogenous kisspeptin secreted by WT and heterozygous littermates present in the same uterus as well as from other maternal tissues. The authors suggested that such 'rescue' was unlikely because, when body weights of the receptor knockout $\left(\mathrm{Kiss} 1 \mathrm{r}^{-/-}\right)$pups, derived from female Kiss1r $r^{+/-}$by male Kiss1r ${ }^{+1-}$ matings, were compared to Kiss $1^{-1-}$ pups, differences were not observed. Because the weights of the Kiss $1 r^{-1-}$ pups could not be rescued in the same manner that Kiss $1^{-/-}$phenotypes could, the authors concluded that this was further evidence that kisspeptin is not required for placental development and function in the mouse.

If KISS1 is not required for placental development in the mouse and rat, it means that trying to better understand the roles of KISS1 in human placentation becomes more challenging. Given the ethical issues associated with studying some aspects of human pregnancy, the use of animal models in preclinical studies is indispensable. However, as the evidence continues to suggest that the commonly used laboratory and livestock species may not be suitable models, researchers must rely more heavily on human cell lines and term placenta and, when possible, placental tissue from early pregnancy voluntary and therapeutic abortions.

\section{Conclusion}

While the importance of KISS1 in regulating human pregnancy is supported through several important observations discussed in this review, limited human clinical data does question this importance. A female patient bearing homozygous L148S mutations in KISS1R and diagnosed with hypogonadotropic hypogonadism and infertility achieved pregnancy through the use of exogenous gonadotropins (Pallais et al. 2006). The first pregnancy, a twin pregnancy, was lost at 6 months of gestation, suggesting that kisspeptin signaling is required for the maintenance of pregnancy. However, this patient, who did not receive kisspeptin or LIF, subsequently carried a singleton pregnancy to term thus leading us to question the significance of kisspeptin in regulating human pregnancy (Pallais et al. 2006). To fully appreciate the importance of kisspeptin in human pregnancy, more female patients with mutations in KISS1 and KISS1R must be identified and a closer examination of their gonadal structure and function, fertility, pregnancy course and delivery will be required.

While this review has focused on the potential roles of peripheral uterine and placental KISS1 in regulating 
pregnancy, it is important to highlight two other recent findings that also likely have a significant impact on pregnancy. In 2014, Tena-Sempere and Ojeda laboratories provided strong evidence for ovarian kisspeptin signaling in folliculogenesis and oocyte maturation and ovulation in the mouse (Dorfman et al. 2014, Gaytan et al. 2014). These findings are of tremendous importance given that oocyte quality affects early embryonic survival and the establishment and maintenance of pregnancy and that quality, or developmental competence, is acquired during folliculogenesis and oocyte maturation. It is likely that these findings will be recapitulated in women given that a recent study showed that KISS1 and KISS1R are differentially expressed in human granulosa and cumulus cells and are co-expressed with NKB/NK3R, providing support for a role of kisspeptin in the regulation of human ovarian function (García-Ortega et al. 2014).

Another important finding comes from Hussain et al. (Song et al. 2014) who reported that in the mouse, hepatic-derived KISS1 acts on $\beta$ cells to suppress glucose-stimulated insulin secretion. They further observed that KISS1 is increased in livers and in serum from humans with type 2 diabetes mellitus (T2DM) and from mouse models of DM. Taken together, it is possible that in a healthy woman, KISS1 might regulate pregnancy both directly and indirectly by regulating insulin levels (Janneau et al. 2002, Horikoshi et al. 2003, Bilban et al. 2004, Hiden et al. 2009, Taylor et al. 2014). As previously discussed, in women KISS1 levels increase with the progression of a pregnancy, and in late pregnancy chronically high KISS1 levels might be protective through the desensitization of the KISS1/ KISS1R signaling system. However, in early pregnancy, prior to this desensitization, KISS1 signaling might be required for implantation and placentation. If that is true, it is easy to appreciate that in women with T2DM, elevated hepatic-derived KISS1 levels might trigger an early desensitization of the KISS1/KISS1R signaling system and thereby disrupt KISS1 signaling required for regulating early pregnancy events.

Clearly, the potential for peripherally derived KISS1 in regulating pregnancy, both directly and indirectly, is significant and warrants detailed investigation. Through such investigations we will not only better understand the roles that KISS1 plays in regulating human fertility and pregnancy but also better appreciate the limitations that animal models present in studying KISS1 in human pregnancy as well as the limitations in using KISS1-based therapies to increase the number and quality of livestock species that benefit from assisted reproduction.

\section{Declaration of interest}

The authors declare that there is no conflict of interest that could be perceived as prejudicing the impartiality of the review.

\section{Funding}

Dr A V Babwah's research is supported by the Natural Sciences and Engineering Research Council of Canada (RGPIN/3273342011).

\section{Acknowledgements}

I would like to sincerely thank Dr Alain Caraty (Physiologie de la Reproduction et des Comportements (INRA/CNRS/Université Tours/Haras Nationaux), 37380 Nouzilly, France) for kindly sharing an abundance of unpublished but highly valuable and reproducible data.

\section{References}

Amenta PS, Gay S, Vaheri A \& Martinez-Hernandez A 1986 The extracellular matrix is an integrated unit: ultrastructural localization of collagen types I, III, IV, V, VI, fibronectin, and laminin in human term placenta. Collagen and Related Research 6 125-152. (doi:10.1016/S0174-173X(86)80021-8)

Arrowsmith S \& Wray S 2014 Oxytocin: its mechanism of action and receptor signalling in the myometrium. Journal of Neuroendocrinology 26 356-369. (doi:10.1111/jne.12154)

Bhatt H, Brunet LJ \& Stewart CL 1991 Uterine expression of leukemia inhibitory factor coincides with the onset of blastocyst implantation. PNAS 88 11408-11412. (doi:10.1073/pnas.88.24.11408)

Bhattacharya M \& Babwah AV 2015 Kisspeptin: beyond the brain. Endocrinology 156 1218-1227. (doi:10.1210/en.2014-1915)

Bilban M, Ghaffari-Tabrizi N, Hintermann E, Bauer S, Molzer S, Zoratti C, Malli R, Sharabi A, Hiden U, Graier W et al. 2004 Kisspeptin-10, a KiSS-1/metastin-derived decapeptide, is a physiological invasion inhibitor of primary human trophoblasts. Journal of Cell Science $\mathbf{1 1 7}$ 1319-1328. (doi:10.1242/jcs.00971)

Boomsma CM, Kavelaars A, Eijkemans MJ, Lentjes EG, Fauser BC, Heijnen CJ \& Macklon NS 2009 Endometrial secretion analysis identifies a cytokine profile predictive of pregnancy in IVF. Human Reproduction 24 1427-1435. (doi:10.1093/humrep/dep011)

Calder M, Chan YM, Raj R, Pampillo M, Elbert A, Noonan M, GillioMeina C, Caligioni C, Berube NG, Bhattacharya M et al. 2014 Implantation failure in female Kiss ${ }^{-1-}$ mice is independent of their hypogonadic state and can be partially rescued by leukemia inhibitory factor. Endocrinology 155 3065-3078. (doi:10.1210/en.2013-1916)

Cejudo Roman A, Pinto FM, Dorta I, Almeida TA, Hernández M, Illanes M, Tena-Sempere M \& Candenas L 2012 Analysis of the expression of neurokinin B, kisspeptin, and their cognate receptors NK3R and KISS1R in the human female genital tract. Fertility and Sterility 97 1213-1219. (doi:10.1016/j.fertnstert.2012.02.021)

Chen JR, Cheng JG, Shatzer T, Sewell L, Hernandez L \& Stewart CL 2000 Leukemia inhibitory factor can substitute for nidatory estrogen and is essential to inducing a receptive uterus for implantation but is not essential for subsequent embryogenesis. Endocrinology 141 4365-4372. (doi:10.1210/endo.141.12.7855)

Cullinan EB, Abbondanzo SJ, Anderson PS, Pollard JW, Lessey BA \& Stewart CL 1996 Leukemia inhibitory factor (LIF) and LIF receptor expression in human endometrium suggests a potential autocrine/paracrine function in regulating embryo implantation. PNAS 93 3115-3120. (doi:10.1073/pnas.93.7.3115)

Dey SK, Lim H, Das SK, Reese J, Paria BC, Daikoku T \& Wang H 2004 Molecular cues to implantation. Endocrine Reviews 25 341-373. (doi:10.1210/er.2003-0020)

Dorfman MD, Garcia-Rudaz C, Alderman Z, Kerr B, Lomniczi A, Dissen GA, Castellano JM, Garcia-Galiano D, Gaytan F, Xu B et al. 2014 Loss of Ntrk2/Kiss1r signaling in oocytes causes premature ovarian failure. Endocrinology 155 3098-3111. (doi:10.1210/en.2014-1111)

Francis VA, Abera AB, Matjila M, Millar RP \& Katz AA 2014 Kisspeptin regulation of genes involved in cell invasion and angiogenesis in first trimester human trophoblast cells. PLoS ONE 9 e99680. (doi:10.1371/ journal.pone.0099680) 
García-Ortega J, Pinto FM, Fernández-Sánchez $M$, Prados N, CejudoRomán A, Almeida TA, Hernández M, Romero $M$, Tena-Sempere $M$ \& Candenas L 2014 Expression of neurokinin B/NK3 receptor and kisspeptin/KISS1 receptor in human granulosa cells. Human Reproduction 29 2736-2746. (doi:10.1093/humrep/deu247)

Gaytan F, Garcia-Galiano D, Dorfman MD, Manfredi-Lozano M, Castellano JM, Dissen GA, Ojeda SR \& Tena-Sempere M 2014 Kisspeptin receptor haplo-insufficiency causes premature ovarian failure despite preserved gonadotropin secretion. Endocrinology 155 3088-3097. (doi:10.1210/en.2014-1110)

Graham CH, Hawley TS, Hawley RG, MacDougall JR, Kerbel RS, Khoo N \& Lala PK 1993 Establishment and characterization of first trimester human trophoblast cells with extended lifespan. Experimental Cell Research 206 204-211. (doi:10.1006/excr.1993.1139)

Groothuis PG, Dassen HH, Romano A \& Punyadeera C 2007 Estrogen and the endometrium: lessons learned from gene expression profiling in rodents and human. Human Reproduction Update 13 405-417. (doi:10.1093/humupd/dmm009)

Grümmer R, Hohn HP, Mareel MM \& Denker HW 1994 Adhesion and invasion of three human choriocarcinoma cell lines into human endometrium in a three-dimensional organ culture system. Placenta $\mathbf{1 5}$ 411-429. (doi:10.1016/0143-4004(94)90008-6)

Hambartsoumian E 1998 Endometrial leukemia inhibitory factor (LIF) as a possible cause of unexplained infertility and multiple failures of implantation. American Journal of Reproductive Immunology 39 137-143. (doi:10.1111/j.1600-0897.1998.tb00345.x)

Herreboudt AM, Kyle VR, Lawrence J, Doran J \& Colledge WH 2015 Kiss1 mutant placentas show normal structure and function in the mouse. Placenta 36 52-58. (doi:10.1016/j.placenta.2014.10.016)

Hiden U, Glitzner E, Hartmann M \& Desoye G 2009 Insulin and the IGF system in the human placenta of normal and diabetic pregnancies. Journal of Anatomy 215 60-68. (doi:10.1111/j.1469-7580.2008.01035.x)

Horikoshi Y, Matsumoto H, Takatsu Y, Ohtaki T, Kitada C, Usuki S \& Fujino M 2003 Dramatic elevation of plasma metastin concentrations in human pregnancy: metastin as a novel placenta-derived hormone in humans. Journal of Clinical Endocrinology and Metabolism 88 914-919. (doi:10.1210/jc.2002-021235)

Janneau JL, Maldonado-Estrada J, Tachdjian G, Miran I, Motte N, Saulnier P, Sabourin JC, Cote JF, Simon B, Frydman R et al. 2002 Transcriptional expression of genes involved in cell invasion and migration by normal and tumoral trophoblast cells. Journal of Clinical Endocrinology and Metabolism 87 5336-5339. (doi:10.1210/jc.2002-021093)

Kimura T, Takemura M, Nomura S, Nobunaga T, Kubota $Y$, Inoue T, Hashimoto K, Kumazawa I, Ito Y, Ohashi K et al. 1996 Expression of oxytocin receptor in human pregnant myometrium. Endocrinology 137 780-785. (doi:10.1210/endo.137.2.8593830)

Kotani $M$, Detheux $M$, Vandenbogaerde A, Communi D, Vanderwinden JM, Le Poul E, Brézillon S, Tyldesley R, SuarezHuerta N, Vandeput F et al. 2001 The metastasis suppressor gene KiSS-1 encodes kisspeptins, the natural ligands of the orphan G proteincoupled receptor KISS1R. Journal of Biological Chemistry 276 34631-34636. (doi:10.1074/jbc.M104847200)

Lee JH, Miele ME, Hicks DJ, Phillips KK, Trent JM, Weissman BE \& Welch DR 1996 KiSS-1, a novel human malignant melanoma metastasissuppressor gene. Journal of the National Cancer Institute 88 1731-1737. (doi:10.1093/jnci/88.23.1731)

Millar RP \& Babwah AV 2015 KISS1R: hallmarks of an effective regulator of the neuroendocrine axis. Neuroendocrinology 101 193-210. (doi:10. 1159/000381457)

Morgan M, Kniss D \& McDonnell S 1998 Expression of metalloproteinases and their inhibitors in human trophoblast continuous cell lines. Experimental Cell Research 242 18-26. (doi:10.1006/excr.1997.3929)

Pallais JC, Bo-Abbas Y, Pitteloud N, Crowley WF Jr \& Seminara SB 2006 Neuroendocrine, gonadal, placental, and obstetric phenotypes in patients with $\mathrm{IHH}$ and mutations in the G-protein coupled receptor, GPR54. Molecular and Cellular Endocrinology 254-255 70-77. (doi:10.1016/j.mce.2006.04.019)

Pampillo M, Camuso N, Taylor JE, Szereszewski JM, Ahow MR, Zajac M, Millar RP, Bhattacharya M \& Babwah AV 2009 Regulation of GPR54 signaling by GRK2 and $\beta$-arrestin. Molecular Endocrinology 23 2060-2074. (doi:10.1210/me.2009-0013)
Pang SC, Janzen-Pang J, Yat Tse M \& Croy BA 2014 The cycling and pregnant mouse: gross anatomy. In The Guide to Investigation of Mouse Pregnancy, pp 3-19. Eds BA Croy, AT Yamada, FJ DeMayo\& SL Adamson. London: Elsevier Academic Press.

Phaneuf S, Europe-Finner GN, Carrasco MP, Hamilton CH \& López Bernal A 1995 Oxytocin signalling in human myometrium. Advances in Experimental Medicine and Biology 395 453-467.

Pinilla L, Aguilar E, Dieguez C, Millar RP \& Tena-Sempere M 2012 Kisspeptins and reproduction: physiological roles and regulatory mechanisms. Physiological Reviews 92 1235-1316. (doi:10.1152/ physrev.00037.2010)

Ramathal CY, Bagchi IC, Taylor RN \& Bagchi MK 2010 Endometrial decidualization: of mice and men. Seminars in Reproductive Medicine 28 17-26. (doi:10.1055/s-0029-1242989)

van der Rest M \& Garrone R 1991 Collagen family of proteins. FASEB Journal 5 2814-2823.

Scott V \& Brown CH 2011 Kisspeptin activation of supraoptic nucleus neurons in vivo. Endocrinology 152 3862-3870. (doi:10.1210/en.20111181)

Scott V \& Brown CH 2013 Beyond the GnRH axis: kisspeptin regulation of the oxytocin system in pregnancy and lactation. Advances in Experimental Medicine and Biology 784 201-218.

Song H \& Lim H 2006 Evidence for heterodimeric association of leukemia inhibitory factor (LIF) receptor and gp130 in the mouse uterus for LIF signaling during blastocyst implantation. Reproduction 131 341-349. (doi:10.1530/rep.1.00956)

Song H, Lim H, Das SK, Paria BC \& Dey SK 2000 Dysregulation of EGF family of growth factors and COX-2 in the uterus during the preattachment and attachment reactions of the blastocyst with the luminal epithelium correlates with implantation failure in LIF-deficient mice. Molecular Endocrinology 14 1147-1161. (doi:10.1210/mend.14.8.0498)

Song WJ, Mondal P, Wolfe A, Alonso LC, Stamateris R, Ong BW, Lim OC, Yang KS, Radovick S, Novaira HJ et al. 2014 Glucagon regulates hepatic kisspeptin to impair insulin secretion. Cell Metabolism 19 667-681. (doi:10.1016/j.cmet.2014.03.005)

Stewart CL, Kaspar P, Brunet LJ, Bhatt H, Gadi I, Köntgen F \& Abbondanzo SJ 1992 Blastocyst implantation depends on maternal expression of leukaemia inhibitory factor. Nature 359 76-79. (doi:10. 1038/359076a0)

Taylor J, Pampillo M, Bhattacharya M \& Babwah AV 2014 Kisspeptin/ KISS1R signaling potentiates extravillous trophoblast adhesion to type-I collagen in a PKC- and ERK1/2-dependent manner. Molecular Reproduction and Development 81 42-54. (doi:10.1002/mrd.22279)

Terao Y, Kumano S, Takatsu Y, Hattori M, Nishimura A, Ohtaki T \& Shintani Y 2004 Expression of KiSS-1, a metastasis suppressor gene, in trophoblast giant cells of the rat placenta. Acta Biochimica et Biophysica Sinica 1678 102-110. (doi:10.1016/j.bbaexp.2004.02.005)

Vogiagis D, Marsh MM, Fry RC \& Salamonsen LA 1996 Leukaemia inhibitory factor in human endometrium throughout the menstrual cycle. Journal of Endocrinology 148 95-102. (doi:10.1677/joe.0.1480095)

Wu S, Zhang H, Tian J, Liu L, Dong Y \& Mao T 2014 Expression of kisspeptin/GPR54 and PIBF/PR in the first trimester trophoblast and decidua of women with recurrent spontaneous abortion. Pathology, Research and Practice 210 47-54. (doi:10.1016/j.prp.2013.09.017)

Xu G, Chakraborty C \& Lala PK 2001 Expression of TGF- $\beta$ signaling genes in the normal, premalignant, and malignant human trophoblast: loss of smad3 in choriocarcinoma cells. Biochemical and Biophysical Research Communications 287 47-55. (doi:10.1006/bbrc.2001.5533)

Zhang P, Tang M, Zhong T, Lin Y, Zong T, Zhong C, Zhang B, Ren M \& Kuang H 2014 Expression and function of kisspeptin during mouse decidualization. PLOS ONE 9 e97647. (doi:10.1371/journal. pone.0097647)

Received 1 June 2015

First decision 6 July 2015

Revised manuscript received 9 July 2015

Accepted 16 July 2015 